\title{
Microvascular complications burden (nephropathy, retinopathy and peripheral polyneuropathy) affects risk of major vascular events and all-cause mortality in type 1 diabetes: a 10-year follow-up study
}

Monia Garofolo ${ }^{1}$, Elisa Gualdani ${ }^{2}$, Rosa Giannarelli ${ }^{1}$, Michele Aragona ${ }^{1}$, Fabrizio Campi ${ }^{1}$, Daniela Lucchesi ${ }^{1}$, Giuseppe Daniele ${ }^{1}$, Roberto Miccoli ${ }^{1}$, Paolo Francesconi ${ }^{2}$, Stefano Del Prato ${ }^{1 *}$ (D) and Giuseppe Penno ${ }^{1}$

\begin{abstract}
Background: Microvascular complications (MC) have been claimed to increase the risk for cardiovascular disease in diabetic subjects. However, the effect of MC burden on the risk of major vascular outcomes and all-cause mortality in type 1 diabetes is still poorly explored. We evaluated the relationship between microvascular complications burden and incidence of major cardiovascular events and all-cause mortality in subjects with type 1 diabetes.

Methods: We recruited 774 participants with type 1 diabetes in a single-center observational study over a follow-up of $10.8 \pm 2.5$ years. Hazard ratios (HR) for cardiovascular outcomes and all-cause death associated with microvascular complications were determined by unadjusted and adjusted Cox regression analysis.

Results: Out of 774 individuals, 54.9\% had no-MC, 32.3\% 1 MC, 9.7\% 2 MC and 3.1\% 3 MC. A total of 54 deaths (7.0\%) occurred. Death rate increased from no-MC 2.1\% (Ref) to 1 MC 7.2\% (HR 3.54 [95\% Cl 1.59-7.87]), 2 MC 14.7\% (HR 6.41 [95\% Cl 2.65-15.49]) and 3 MC 66.7\% (HR 41.73 [95\% Cl 18.42-94.57], $\mathrm{p}<0.0001$ ). After adjustments, HRs were: $1 \mathrm{MC}$ 2.05 (95\% Cl 0.88-4.76), 2 MC 1.98 (95\% Cl 0.75-5.21), 3 MC 7.02 (95\% Cl 2.44-20.20, p=0.002). Forty-nine subjects (6.7\%) had at least one cardiovascular event, and cumulative incidence went from no-MC 2.2\% (Ref) to $1 \mathrm{MC}$ 5.0\%; (HR 2.27 [95\% Cl 0.96-5.38]), 2 MC 26.8\% (HR 12.88 [95\% Cl 5.82-28.50]) and 3 MC 40.9\% (HR 29.34 [95\% Cl 11.59-74.25], $\mathrm{p}<0.0001$ ). Upon adjustments, HRs were: 1 MC 1.59 (95\% Cl 0.65-3.88), 2 MC 4.33 (95\% Cl 1.75-10.74), 3 MC 9.31 (95\% $\mathrm{Cl} 3.18-27.25, \mathrm{p}<0.0001)$. Thirty-five individuals (4.8\%) had at least one coronary event, which cumulative incidence increased with MC burden $(p<0.0001)$.
\end{abstract}

Conclusions: In type 1 diabetes, microvascular complications burden increases in an independent dose-dependent manner the risk of major cardiovascular outcomes and all-cause mortality. The presence and number of microvascular complications should be considered in stratifying overall cardiovascular risk in type 1 diabetes.

Keywords: Type 1 diabetes mellitus, Microvascular complications, Microvascular burden, Diabetic kidney disease, Diabetic retinopathy, Peripheral diabetic polyneuropathy, All-cause mortality, Cardiovascular disease

\footnotetext{
*Correspondence: stefano.delprato@med.unipi.it

1 Section of Diabetes and Metabolic Disease, Department of Clinical and Experimental Medicine, University of Pisa and Azienda Ospedaliero-

Universitaria Pisana, Via Paradisa, 2, 56124 Pisa, Italy

Full list of author information is available at the end of the article
} 


\section{Background}

In spite of recent reports suggesting reduction of mortality rate among subjects with type 1 diabetes [1], mortality still remains higher compared to the general population [1] as recently reported in North America [2, 3], Europe [4-6] and Australia [7]. Petrie et al. reported that Swedish individuals with type 1 diabetes have a life expectancy (LE) gap at age 20 of about 10-12 years compared with the general population [6]. Consistently, Huo et al. show that, at birth, Australian subjects with type 1 diabetes have an estimated LE loss of 12.2 years compared with those without diabetes [7].

This excess risk persists even if all cardiovascular $(\mathrm{CV})$ risk factors are properly controlled [8]. In particular it has been calculated that the risk of all-cause as well as cardiovascular death in type 1 diabetic subjects with good glycaemic control remains twice as much the risks in the general population [9].

These findings highlight the need to improve risk stratification among type 1 diabetic subjects in the attempt to identify those with very high risk. To this purpose, risk prediction models based on easily accessible patient's clinical characteristics have been proposed $[10,11]$.

Epidemiological studies suggest that the presence of microvascular complications can increase the risk of $\mathrm{CV}$ disease and overall mortality in type 1 diabetes $[12,13]$. In the Joslin 50-year Medalist Study and in the Finnish Diabetic Nephropathy Study (FinnDiane) the coexistence of proliferative diabetic retinopathy (PDR) and stage $\geq 3 \mathrm{~b}$ diabetic kidney disease (DKD) was associated with higher prevalence of $\mathrm{CV}$ disease in patients with longstanding type 1 diabetes [14]. Nonetheless, till recently [15], cross-sectional [14, 16] and prospective data [17] assessing the impact of cumulative microvascular disease burden on CV disease and overall mortality in type 1 diabetes have been scanty.

Therefore, we have investigated to which extent the cumulative burden of retinopathy, nephropathy, and peripheral neuropathy is associated with the incidence of all-cause mortality, major CV disease and coronary heart disease (CHD) irrespective of conventional CV risk factors in individuals with type 1 diabetes. Our hypothesis is that the increasing microvascular complication burden, i.e. the number of microvascular complications, can increase the risk of all-cause mortality and incidence of $\mathrm{CV}$ events, being the highest in those with complications in all three microvascular beds.

\section{Methods}

\section{Study design and population}

This is an observational, cross-sectional, single-centre study with baseline $\mathrm{CV}$ risk stratification and prospective assessment of all-cause mortality and major vascular outcomes in a cohort of individuals with type 1 diabetes. The research design and study population have been previously described [18]. All subjects $(n=843)$ attending the Diabetes Outpatient Clinic of the Azienda Ospedaliero-Universitaria Pisana from January 1, 2001 to December 31, 2009 for their usual screening for diabetic complications were considered eligible. Type 1 diabetes was diagnosed on the basis of age at diagnosis $<36$ years, immediate requirement of insulin therapy and unbroken need for insulin therapy for the first year after diagnosis [19]. Pregnant women, participants of not-white ethnicity, those with diabetes duration $<1$ year, those on dialysis or with renal transplantation, and three individuals for whom valid information on vital status could not be retrieved were excluded. Finally, a total of 774 individuals $(52.6 \%$ men; age $40.2 \pm 11.7$ years; duration of diabetes $19.4 \pm 12.2$ years, mean $\pm S D$ ) entered the study. The Ethics Committee of the University of Pisa approved the study protocol, consent procedures, and data analysis plan.

Information about onset and duration of diabetes (DD), smoking habits, insulin therapy, and blood pressure- and lipid-lowering therapies were collected for all subjects along with body mass index (BMI), waist-to-hip ratio (WHR), and resting blood pressure (BP). Hypertension was defined as systolic $\mathrm{BP}>140 \mathrm{mmHg}$ and/or diastolic $\mathrm{BP}>80 \mathrm{mmHg}$ and/or use of antihypertensive medications.

Serum creatinine, glycated haemoglobin $\left(\mathrm{HbA}_{1 \mathrm{c}}\right)$, total- and HDL-cholesterol, triacylglycerol, alanine aminotransferase (ALT), aspartate aminotransferase (AST), gamma-glutamyltransferase (GGT), uric acid and fibrinogen were determined in all subjects at the time of first screening for diabetic complications.

\section{Baseline measurements}

Albumin-to-creatinine ratio (ACR) was determined in at least three first-voided urine samples obtained with at least 1-month interval in the year preceding the recruitment. Urine samples $(n=175,6.7 \%)$ with abnormal sediment (nitrites or $\geq 250$ leucocytes $/ \mathrm{ml}$ ) were discarded. All other samples were assayed for albumin (BNII; Dade Behring Diagnostic, Marburg, Germany; intra- and interassay variation $<2.0 \%$ and $<3.5 \%$, respectively) and creatinine (modified Jaffé reaction) on the same morning of collection.

$\mathrm{HbA}_{1 \mathrm{c}}$ was measured by high-performance liquid chromatography using DCCT-aligned methods [20]; triacylglycerol, total- and HDL-cholesterol were determined by colorimetric enzymatic methods, and LDLcholesterol was calculated by the Friedewald formula [21]. Standard clinical laboratory methods have been 
employed for the measurement of serum creatinine, ALT, AST, GGT, uric acid and fibrinogen.

\section{Assessment of diabetes complications}

Diabetic kidney disease was established based on the geometric mean of three ACR values, and estimated glomerular filtration rate (eGFR) was calculated by the Chronic Kidney Disease Epidemiology Collaboration (CKD-EPI) equation [22]. DKD was diagnosed on the basis of ACR $>3.4 \mathrm{mg} / \mathrm{mmol}$ [23] and/or $\mathrm{eGFR}<60 \mathrm{~mL} / \mathrm{min} / 1.73 \mathrm{~m}^{2}$ [24].

The presence and severity of diabetic retinopathy (DR) was assessed by retinal photography using a wide-angle $\left(45^{\circ}\right)$ midriatic camera. Photos of discmacula-temporal and disc-nasal regions were taken [25] and the eye with worse retinal condition including previous photocoagulation or surgical treatment, was used for retinopathy staging: absent, mild, moderate or severe non-proliferative, proliferative diabetic retinopathy, or maculopathy, according to the Global Diabetic Retinopathy Project Group criteria [26]. For further analysis, individuals with non-proliferative retinopathy of mild or moderate degree were classified as nonadvanced diabetic retinopathy, whereas those with severe non-proliferative, proliferative, maculopathy, or blindness were grouped into the advanced, sightthreatening diabetic retinopathy category.

Diabetic peripheral neuropathy was defined on the presence of symptoms and signs assessed by the Michigan Neuropathy Screening Instrument questionnaire designed to identify symptoms of muscle weakness and sensory dysfunction [19,27], by the examination of the feet for neuropathic ulcerations, by reduced or absent knee and ankle reflexes, and/or by measurement of vibration perception threshold using a biothesiometer applied bilaterally at the medial malleolus and the tip of the big toe $[19,27]$. The mean of three readings at $10 \mathrm{~s}$ intervals on each site was calculated and interpreted according to age-specific reference values [28].

Presence of previous CV disease was ascertained on the basis of medical history of any major acute $\mathrm{CV}$ events, i.e. myocardial infarction, stroke, ischemic foot ulcer or gangrene, amputation and coronary, carotid, and/or lower limb revascularization. A 12 lead resting ECG was recorded and coded according to the Minnesota Code [29]. Peripheral vascular disease was defined on a positive history of ischemic ulceration, gangrene, amputation or lower limb revascularization, or diagnosed on the presence of reduced or absent femoral and/or foot pulses and reduced ankle/brachial pressure ratio $(<0.9)$.

\section{Calculation of the EURODIAB PCS risk score}

The EURODIAB Prospective Complications Study (PCS) risk score was calculated as proposed by SoedamahMuthu et al. [10]. The score calculates risk for major outcomes (CHD, stroke, end stage renal disease, amputation, blindness) and all-cause death in type 1 diabetes on the basis of age, $\mathrm{HbA}_{1 \mathrm{c}}$, WHR, ACR, and HDL levels allowing risk stratification as low (LS, $\leq 15)$, intermediate (IS, 16 to $19)$ and high score (HS, $\geq 20$ ).

\section{Assessment of outcomes}

The primary outcome was the time to all-cause mortality. Follow-up data for each patients were verified up to December 31, 2017. Vital status was available for all participants and was verified over a mean follow-up of $10.8 \pm 2.5$ years (median 10.2, IQR 9.2-12.3) by interrogation of the Italian Health Card Database (http://siste mats1.sanita.finanze.it/wps/portal/). The secondary outcomes were the time to first major CV event (i.e. occurrence of first event of myocardial infarction, coronary revascularization, stroke, carotid revascularization, and ulcer, gangrene, amputation and peripheral revascularization) and the time to first coronary event (i.e. occurrence of first event of myocardial infarction or coronary revascularization). Data for major $\mathrm{CV}$ and coronary events were available for 736 participants (95.1\%), and were obtained, upon data anonymization (in compliance with current privacy legislation), in collaboration with ARS Toscana (Regional Health Agency of the Tuscany Region) through hospital discharge registers. ICD-9-CM codes (International Classification of Diseases, 9th Edition, Clinical Modification) was used to detect major CV and coronary artery events (Additional file 1: Table S1). All events (for primary and secondary outcomes) occurring between date of enrollment and end of follow-up or death were considered as incident.

\section{Statistical analyses}

Data are expressed as median (interquartile range, IQR) and/or mean $\pm S D$ for continuous variables, and number of cases and percentage for categorical variables. Continuous variables were compared by the Student's t-test or one-way ANOVA (Welch robust test for equality of means when appropriate based on the Levene statistic) for normally distributed variables. Wilcoxon Sum-ofRanks (Mann-Whitney) U test or Kruskal-Wallis tests were used for variables with skewed distribution. Pearson $x^{2}$ or the Fisher exact tests were applied to categories. The Spearman's rank-order correlation was run to measure strength and direction of associations between two variables measured on ordinal scale. For post hoc comparisons, Scheffe's test or Tamhane's test, Mann-Whitney 
$\mathrm{U}$ test and $\mathrm{X}^{2}$ test were used for normally distributed, not-normally distributed, and categorical variables, respectively.

Crude mortality rates and incidence of outcomes were described as events per 1000 person-years (PYs), with 95\% exact Poisson Confidence Intervals (CI). Time to allcause death or to each first outcome was plotted according to the MC groups as Kaplan-Meier (K-M) curves, and comparison was made using the log rank test. Univariate and multivariate Cox proportional hazard models were used to identify the effect of key covariates such as sex and age (model 1), or sex, age and cardiovascular risk factors (model 2), and sex, cardiovascular risk factors and the EURODIAB PCS risk score (model 3). Results are expressed as Hazard Ratio (HR) and 95\% CI. Analyses have been performed in the whole cohort as well as in subjects free of $\mathrm{CV}$ disease at baseline (CVD-). A two-sided $\mathrm{p}$ value $\leq 0.05$ was considered statistically significant.

A post hoc power calculation has been performed to evaluate the statistical power of our sample size for the primary outcome, i.e. all-cause mortality. Compared to the reference group (subject without $\mathrm{MC}$ ), our groups of subjects with 1, 2 and $3 \mathrm{MC}$ have a post hoc power of $87.5 \%, 97 \%$ and $100 \%$, respectively, to detect the observed differences in mortality rate with an alpha error level of $5 \%$.

All statistical analyses were performed using SPSS package 25.0 version (IBM SPSS, Chicago, IL).

\section{Results}

We recruited 774 individuals with type 1 diabetes who have been followed up to a mean of $10.8 \pm 2.5$ years equivalent to 8387 PYs follow-up. At screening, 425 participants (54.9\%) had no-MC, 250 (32.3\%) had $1 \mathrm{MC}$, 75 (9.7\%) $2 \mathrm{MC}$, and 24 (3.1\%) $3 \mathrm{MC}$ (Additional file 1: Figure S1). Distribution was unchanged $(\mathrm{n}=418,57.0 \%$; $\mathrm{n}=236,32.2 \% ; \mathrm{n}=62,8.5 \%$ and $\mathrm{n}=17,2.3 \%$, respectively; $\mathrm{p}=0.620)$ upon exclusion of 41 subjects $(5.3 \%)$ with prior CV events (CVD+).

Table 1 and Additional file 1: Table S2 show the baseline characteristics of the whole cohort. Overall the presence and severity of $\mathrm{MC}$ were associated with age, diabetes duration, $\mathrm{BMI}$ and $\mathrm{WHR}, \mathrm{HbA}_{1 \mathrm{c}}$, systolic and diastolic BP, prevalence of hypertension, and rates of treatment with BP-lowering agents and renin-angiotensin system (RAS) blockers. On the contrary, there was no difference in gender distribution, smoking history, HDLcholesterol, and triacylglycerol. Total- and LDL-cholesterol tended to be lower in individuals with $3 \mathrm{MC}$, most likely because of more common use of lipid-lowering therapy. MC burden was also associated with increasing levels of non-conventional risk factors such as uric acid and fibrinogen, lower eGFR and higher ACR ( $\mathrm{p}<0.0001$ for all). Finally, the rate of previous $\mathrm{CV}$ events and prevalence of high-risk EURODIAB PCS score $(\geq 20)$ also increased with $\mathrm{MC}$ ( $\mathrm{p}<0.0001$ for both).

\section{Microvascular complications and all-cause mortality in the whole cohort}

During study follow-up 54 individuals (7.0\%; $6.44 \times 1000$ PYs) died. Mortality increased with MC $(\mathrm{p}<0.0001)$ going from $2.1 \%$ in subjects with no-MC $(n=9$; $1.92 \times 1000$ PYs; Ref), to $7.2 \%$ in those with $1 \mathrm{MC}(\mathrm{n}=18$; $6.76 \times 1000$ PYs; HR 3.54 [95\% CI 1.59-7.87]), 14.7\% in those with $2 \mathrm{MC}(\mathrm{n}=11 ; 12.93 \times 1000$ PYs; HR 6.41 [95\% CI 2.65-15.49]), and 66.7\% in individuals with 3 MC $(n=16 ; 79.77 \times 1000$ PYs; HR 41.73 [95\% CI 18.4294.57]; Fig. 1a). After adjustment for confounders (Fig. 2, Models 1 to 3 ), the HRs for primary outcome remained significant with the risk being the highest in those with 3 MC. In particular, in Model 3, HRs for death went from 2.05 in $1 \mathrm{MC}(95 \% \mathrm{CI} 0.88-4.76)$, to 1.98 (95\% CI $0.75-$ 5.21 ) in $2 \mathrm{MC}$, and 7.02 (95\% CI 2.44-20.20, $\mathrm{p}=0.002$ ) in $3 \mathrm{MC}$, with independent effects for active smoking, triacylglycerol, uric acid and intermediate- (HR 3.08 [95\% CI 1.25-7.56]) and high-risk EURODIAB PCS score (HR 8.59 [95\% CI 3.40-21.71], p < 0.0001).

\section{Microvascular complications and all-cause mortality in subjects free of CV disease at baseline (CVD-)}

Death rate increased with MC also in CVD- $(n=733$; $\mathrm{p}<0.0001)$. At follow-up 43 subjects $(5.9 \%)$ died: $2.2 \%$ in no-MC $(\mathrm{n}=9 ; 1.95 \times 1000 \mathrm{PYs}), 6.4 \%$ in $1 \mathrm{MC}(\mathrm{n}=15$; $5.95 \times 1000 \mathrm{PYs}), 12.9 \%$ in $2 \mathrm{MC}(\mathrm{n}=8 ; 11.30 \times 1000$ $\mathrm{PYs})$, and $64.7 \%$ in $3 \mathrm{MC}(\mathrm{n}=11 ; 72.00 \times 1000$ PYs; Additional file 1: Figure S2). In CVD-, after adjustments (Fig. 3, Models 1 to 3), the effects of MC on all-cause mortality remained significant up to Model 3, where the $\mathrm{HR}$ for $3 \mathrm{MC}$ was 5.46 (95\% CI 1.67-17.86, p=0.005) independently of uric acid and the EURODIAB PCS score.

\section{Microvascular complications and incidence of major cardiovascular and coronary events in the whole cohort} Major CV events occurred in 49 out of 736 subjects (6.7\%; $6.42 \times 1000$ PYs) during a mean follow-up of $10.4 \pm 2.9$ years. Incidence of major $\mathrm{CV}$ events increased with MC ( $<<0.0001)$ : no-MC 2.2\% ( $\mathrm{n}=9 ; 2.09 \times 1000$ PYs; Ref), 1 MC 5.0\% ( $=12 ; 4.79 \times 1000$ PYs; HR 2.27 [95\% CI 0.96-5.38]), 2 MC 26.8\% ( $\mathrm{n}=19 ; 27.56 \times 1000$ PYs; HR 12.88 [95\% CI 5.82-28.50]), 3 MC 40.9\% ( $\mathrm{n}=9$; $63.82 \times 1000$ PYs; HR 29.34 [95\% CI 11.59-74.25]; Fig. 1b). In adjusted Cox regression analysis (Fig. 4a, Models 1 to 3 ), the risk for major CV events, compared to no-MC, increased in subjects with $2 \mathrm{MC}$ and more so 
Table 1 Baseline characteristics of the study cohort, both overall and according to microvascular complications burden

\begin{tabular}{|c|c|c|c|c|c|c|}
\hline & $\begin{array}{l}\text { All patients } \\
(n=774)\end{array}$ & No MC $(n=425)$ & $1 \mathrm{MC}(\mathrm{n}=250)$ & $2 M C(n=75)$ & $3 \mathrm{MC}(\mathrm{n}=24)$ & $p$ value \\
\hline Men/women, n (\%) & $407 / 367(52.6 / 47.4)$ & $224 / 201(52.7 / 47.3)$ & $130 / 120(52.0 / 48.0)$ & 36/39 (48.0/52.0) & $17 / 7(70.8 / 29.2)$ & 0.275 \\
\hline Age, years & $40.2 \pm 11.7$ & $36.3 \pm 10.1$ & $43.0 \pm 10.4^{* * *}$ & $48.4 \pm 13.0^{* * *+\dagger}$ & $55.7 \pm 13.7^{* * * \text { 十†† }}$ & $<0.0001$ \\
\hline $\begin{array}{l}\text { Age at diabetes } \\
\text { diagnosis, years }\end{array}$ & $20.8 \pm 10.9$ & $23.2 \pm 10.7$ & $17.5 \pm 10.0^{* * *}$ & $17.7 \pm 11.6^{* * *}$ & $24.6 \pm 8.9^{\dagger \ddagger}$ & $<0.0001$ \\
\hline $\begin{array}{l}\text { Duration of diabetes, } \\
\text { years }\end{array}$ & $19.4 \pm 12.2$ & $13.1 \pm 9.4$ & $25.5 \pm 10.2^{* * *}$ & $30.7 \pm 11.2^{* * *+\dagger+}$ & $31.1 \pm 12.9^{* * *}$ & $<0.0001$ \\
\hline $\mathrm{BMI}, \mathrm{kg} / \mathrm{m}^{2}$ & $24.8 \pm 3.6$ & $24.2 \pm 3.3$ & $25.3 \pm 3.7^{* * *}$ & $26.2 \pm 3.7^{* * *}$ & $26.8 \pm 3.9^{* *}$ & $<0.0001$ \\
\hline WHR & $0.921 \pm 0.063$ & $0.914 \pm 0.056$ & $0.924 \pm 0.069$ & $0.943 \pm 0.070^{* *}$ & $0.945 \pm 0.081$ & $<0.0001$ \\
\hline $\begin{array}{l}\text { Smoking habits (non- } \\
\text { smokers, current } \\
\text { smokers) (n. 759), } \\
\text { n (\%) }\end{array}$ & $534 / 225(70.4 / 29.6)$ & 295/121 (70.9/29.1) & $168 / 78(68.3 / 31.7)$ & 53/21 (71.6/28.4) & $18 / 5(78.3 / 21.7)$ & 0.727 \\
\hline $\begin{array}{l}\text { Fasting glucose, } \\
\mathrm{mmol} / \mathrm{L}\end{array}$ & $9.44 \pm 4.56$ & $9.10 \pm 4.23$ & $9.44 \pm 4.78$ & $10.74 \pm 5.18^{*}$ & $11.44 \pm 4.80$ & 0.005 \\
\hline $\begin{array}{l}\mathrm{HbA}_{1 \mathrm{c}} \%(\mathrm{mmol} / \\
\mathrm{mol})\end{array}$ & $\begin{array}{l}7.83 \pm 1.18 \\
(62.1 \pm 12.9)\end{array}$ & $\begin{array}{l}7.70 \pm 1.19 \\
(60.6 \pm 13.0)\end{array}$ & $\begin{array}{l}7.85 \pm 1.11 \\
\quad(62.3 \pm 12.2)\end{array}$ & $\begin{array}{l}8.25 \pm 1.10^{* *} \\
(66.7 \pm 12.0)\end{array}$ & $\begin{array}{r}8.60 \pm 1.30^{* * \dagger} \\
(70.4 \pm 14.2)\end{array}$ & $<0.0001$ \\
\hline Systolic BP, mmHg & $127 \pm 18$ & $122 \pm 15$ & $130 \pm 18^{* * *}$ & $136 \pm 20^{* * *+}$ & $150 \pm 16^{* * * 十+十 \text { 护 }}$ & $<0.0001$ \\
\hline Diastolic BP, mmHg & $73 \pm 9$ & $72 \pm 8$ & $75 \pm 9^{* *}$ & $74 \pm 10$ & $79 \pm 11^{* *}$ & $<0.0001$ \\
\hline $\begin{array}{l}\text { Total cholesterol, } \\
\mathrm{mmol} / \mathrm{L}\end{array}$ & $4.84 \pm 0.88$ & $4.72 \pm 0.85$ & $5.00 \pm 0.90^{* *}$ & $5.08 \pm 0.85^{*}$ & $4.68 \pm 1.07$ & $<0.0001$ \\
\hline $\begin{array}{l}\text { LDL cholesterol, } \\
\mathrm{mmol} / \mathrm{L}\end{array}$ & $3.01 \pm 0.76$ & $2.94 \pm 0.76$ & $3.11 \pm 0.74^{*}$ & $3.12 \pm 0.73$ & $2.92 \pm 0.94$ & 0.019 \\
\hline \multicolumn{7}{|l|}{ HDL cholesterol, mmol/L } \\
\hline Men & $1.45(1.24-1.71)$ & $1.44(1.22-1.71)$ & $1.53(1.29-1.71)$ & $1.49(1.32-1.68)$ & $1.40(1.06-1.73)$ & 0.644 \\
\hline Women & $1.74(1.48-2.07)$ & $1.71(1.42-1.99)$ & $1.79(1.51-2.15)$ & $1.79(1.58-2.15)$ & $1.76(1.37-1.84)$ & 0.246 \\
\hline \multicolumn{7}{|c|}{ Triacylglycerol, mmol/L } \\
\hline Men & $0.90(0.71-1.24)$ & $0.86(0.69-1.15)$ & $0.94(0.71-1.30)$ & $1.00(0.81-1.68)$ & $1.22(0.81-1.62)$ & 0.031 \\
\hline Women & $0.78(0.60-1.06)$ & $0.75(0.58-1.04)$ & $0.80(0.63-1.06)$ & $0.84(0.66-1.06)$ & $1.32(0.80-1.33)$ & 0.706 \\
\hline $\mathrm{ALT}, \mathrm{U} / \mathrm{L}$ & $20.0 \pm 10.8$ & $19.7 \pm 11.5$ & $20.1 \pm 9.0$ & $20.6 \pm 8.9$ & $23.6 \pm 18.7$ & 0.343 \\
\hline AST, U/L & $22.3 \pm 31.8$ & $20.9 \pm 16.1$ & $24.4 \pm 27.6$ & $21.6 \pm 10.9$ & $26.7 \pm 38.2$ & 0.495 \\
\hline Gamma-GT, U/L & $20.6 \pm 33.3$ & $18.4 \pm 27.9$ & $20.2 \pm 20.5$ & $22.5 \pm 18.0$ & $56.2 \pm 125.8^{* * *+十 \dagger \neq \neq \neq}$ & $<0.0001$ \\
\hline Uric acid, $\mu \mathrm{mol} / \mathrm{L}$ & $223.6 \pm 67.4$ & $213.7 \pm 58.3$ & $241.4 \pm 72.3$ & $316.3 \pm 102.0^{* * *+t \dagger}$ & $381.7 \pm 152.1^{* * *+十 \dagger き}$ & $<0.0001$ \\
\hline Fibrinogen, $\mu \mathrm{mol} / \mathrm{L}$ & $9.89 \pm 2.00$ & $9.63 \pm 1.93$ & $10.93 \pm 2.33$ & $10.77 \pm 1.59^{* * *+\dagger}$ & $12.32 \pm 2.56^{* * *+\dagger}$ & $<0.0001$ \\
\hline Creatinine, $\mu \mathrm{mol} / \mathrm{L}$ & $73.3 \pm 18.6$ & $70.8 \pm 12.8$ & $72.0 \pm 12.6$ & $79.6 \pm 24.9^{* * * \dagger}$ & $111.3 \pm 55.5^{* * * \text { 十†† まキ }}$ & $<0.0001$ \\
\hline $\begin{array}{l}\text { Albumin-to-creati- } \\
\text { nine ratio (ACR), } \\
\text { mg/mmol }\end{array}$ & $0.49(0.26-1.00)$ & $0.40(0.23-0.75)$ & $0.52(0.26-0.97)$ & $1.66^{* * *++\dagger}(0.40-8.38)$ & $\begin{array}{l}8.31^{* * *+十+\neq \neq \neq} \\
(3.67-51.08)\end{array}$ & $<0.0001$ \\
\hline $\begin{array}{l}\text { eGFR, CKD-EPI, mL/ } \\
\mathrm{min} / 1.73 \mathrm{~m}^{2}\end{array}$ & $102.5 \pm 17.4$ & $107.4 \pm 14.1$ & $101.1 \pm 13.8^{* * *}$ & $90.4 \pm 21.9^{* * *+\dagger}$ & $68.9 \pm 28.0^{* * *+十 † \neq \neq \ddagger ~}$ & $<0.0001$ \\
\hline $\begin{array}{l}\text { Daily insulin dose, IU/ } \\
\text { kg body weight }\end{array}$ & $0.66 \pm 0.20$ & $0.66 \pm 0.21$ & $0.67 \pm 0.19$ & $0.66 \pm 0.22$ & $0.78 \pm 0.21$ & 0.070 \\
\hline MDI/CSII, n (\%) & $691 / 83(89.3 / 10.7)$ & $380 / 45(89.4 / 10.6)$ & 223/27 (89.2/10.8) & 65/10 (86.7/13.3) & 23/1 (95.8/4.2) & 0.655 \\
\hline $\begin{array}{l}\text { Treatment with BP- } \\
\text { lowering agents, } \\
\text { n (\%) }\end{array}$ & $151(19.5)$ & $28(6.6)$ & $61(24.4)^{* * *}$ & $46(61.3)^{* * *+t+}$ & $16(66.7)^{* * *+t+}$ & $<0.0001$ \\
\hline $\begin{array}{l}\text { Treatment with RAS } \\
\text { blockers, n (\%) }\end{array}$ & $136(17.6)$ & $23(5.4)$ & $56(22.4)^{* * *}$ & $42(56.0)^{* * *+t \dagger}$ & $15(62.5)^{* * *+t+}$ & $<0.0001$ \\
\hline $\begin{array}{l}\text { Treatment with lipid- } \\
\text { lowering agents, } \\
\text { n (\%) }\end{array}$ & $100(12.9)$ & $35(8.2)$ & $38(15.2)^{* *}$ & $18(24.0)^{* * *}$ & $9(37.5)^{* * *+\dagger}$ & $<0.0001$ \\
\hline $\begin{array}{l}\text { Treatment with } \\
\text { antiplatelet drugs, } \\
\mathrm{n}(\%)\end{array}$ & $50(6.5)$ & $6(1.4)$ & $19(7.6)^{* * *}$ & $17(22.7)^{* * *+t \dagger}$ & $8(33.3)^{* * *+t+}$ & $<0.0001$ \\
\hline
\end{tabular}


Table 1 (continued)

\begin{tabular}{|c|c|c|c|c|c|c|}
\hline & $\begin{array}{l}\text { All patients } \\
(n=774)\end{array}$ & No MC $(n=425)$ & $1 \mathrm{MC}(\mathrm{n}=250)$ & $2 M C(n=75)$ & $3 M C(n=24)$ & $p$ value \\
\hline $\begin{array}{l}\text { Treatment with met- } \\
\text { formin, } \mathrm{n}(\%)\end{array}$ & $46(5.9)$ & $18(4.2)$ & $20(8.0)$ & $5(6.7)$ & $3(12.5)$ & 0.110 \\
\hline Hypertension, n (\%) & $270(34.9)$ & $82(19.3)$ & $110(44.0)^{* * *}$ & $55(73.3)^{* * *+十 \dagger}$ & $23(95.8)^{* * * \text { 十†† }}$ & $<0.0001$ \\
\hline $\begin{array}{l}\text { Retinopathy: no } \\
\text { retinopathy/ } \\
\text { non advanced/ } \\
\text { advanced, n (\%) }\end{array}$ & $\begin{array}{l}452 / 202 / 120 \\
(58.4 / 26.1 / 15.5)\end{array}$ & $425 / 0 / 0(100 / 0 / 0)$ & $\begin{array}{l}26 / 164 / 60^{* * *} \\
(10.4 / 65.6 / 24.0)\end{array}$ & $\begin{array}{l}1 / 32 / 42^{* * *+t \dagger} \\
(1.3 / 42.7 / 56.0)\end{array}$ & $\begin{array}{l}\text { O/6/18***t+ } \\
(0 / 25.0 / 75.0)\end{array}$ & $<0.0001$ \\
\hline $\begin{array}{l}\text { Peripheral polyneu- } \\
\text { ropathy: no/yes, } \\
\text { n (\%) }\end{array}$ & 706/68 (91.2/8.8) & $425 / 0(100 / 0)$ & $241 / 9 * * *(96.4 / 3.6)$ & $\begin{array}{r}40 / 35^{* * *+t+\dagger} \\
(53.3 / 46.7)\end{array}$ & $0 / 24^{* * *}$ †十†抹 $(0 / 100)$ & $<0.0001$ \\
\hline $\begin{array}{l}\text { Diabetic kidney dis- } \\
\text { ease: no/yes, n (\%) }\end{array}$ & 792/82 (89.4/10.6) & $425 / 0(100 / 0)$ & $233 / 17^{* * *}(93.2 / 6.8)$ & $\begin{array}{r}34 / 41^{* * *+t+} \\
(45.3 / 54.7)\end{array}$ & $0 / 24^{* * *+十 \dagger \neq \neq \neq}(0 / 100)$ & $<0.0001$ \\
\hline $\begin{array}{l}\text { Major adverse car- } \\
\text { diovascular events, } \\
\mathrm{n}(\%)\end{array}$ & $41(5.3)$ & $7(1.6)$ & $14(5.6)^{* *}$ & $13(17.3)^{* * *+\dagger}$ & $7(29.2)^{* * *++\dagger}$ & $<0.0001$ \\
\hline $\begin{array}{l}\text { Coronary artery } \\
\text { disease, } \mathrm{n}(\%)\end{array}$ & $29(3.7)$ & $3(0.7)$ & $10(4.0)^{* *}$ & $10(13.3)^{* * *+\dagger}$ & $6(25.0)^{* * *+1}$ & $<0.0001$ \\
\hline Stroke, n (\%) & $4(0.5)$ & $2(0.5)$ & $0(0)$ & $2(2.7)$ & $0(0)$ & 0.090 \\
\hline $\begin{array}{l}\text { EURODIAB PCS risk } \\
\text { score: LS, IS, HS, } \\
\text { n (\%) }\end{array}$ & $\begin{array}{l}466 / 205 / 103 \\
(60.2 / 26.5 / 13.3)\end{array}$ & $\begin{array}{l}324 / 84 / 17 \\
(76.2 / 19.8 / 4.0)\end{array}$ & $\begin{array}{l}127 / 87 / 36^{* * *} \\
(50.8 / 34.8 / 14.4)\end{array}$ & $\begin{array}{l}14 / 30 / 31^{* * *++\dagger} \\
(18.7 / 40.0 / 41.3)\end{array}$ & $\begin{array}{l}\text { 1/4/19***十+十执 } \\
(4.2 / 16.7 / 79.2)\end{array}$ & $<0.0001$ \\
\hline Cancer, n (\%) & $9(1.2)$ & $3(0.7)$ & $2(0.8)$ & $3(4.0)^{*}$ & $1(4.2)$ & 0.042 \\
\hline $\begin{array}{l}\text { Autoimmune thyre- } \\
\text { opathy, n (\%) }\end{array}$ & $113(14.6)$ & $58(13.6)$ & $44(17.6)$ & $8(10.7)$ & $3(12.5)$ & 0.372 \\
\hline
\end{tabular}

Quantitative variables are shown as mean \pm SD or median (IQR)

$\mathrm{MDI} / \mathrm{CS} / /$ multiple daily insulin injections/continuous subcutaneous insulin infusion

${ }^{*} \mathrm{p}<0.05$; ${ }^{* *} \mathrm{p}<0.01$; ${ }^{* *} \mathrm{p}<0.001$ vs no $\mathrm{MC}$

${ }^{+} \mathrm{p}<0.05 ;{ }^{{ }^{+\dagger}} \mathrm{p}<0.01 ;{ }^{+{ }^{+\dagger}} \mathrm{p}<0.001$ vs $1 \mathrm{MC}$

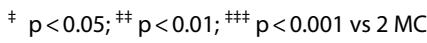

in those with $3 \mathrm{MC}$. In particular, in Model 3, HRs were: 1 MC 1.59 (95\% CI 0.65-3.88), 2 MC 4.33 (95\% CI 1.7510.74), 3 MC 9.31 (95\% CI 3.18-27.25, p<0.0001) with independent effects for fibrinogen, hypertension, and prior CV events.

Coronary events occurred in 35 out of 736 subjects (4.8\%; $4.54 \times 1000 \mathrm{PYs})$ during a mean follow-up of $10.5 \pm 2.7$ years with incidence increasing with $\mathrm{MC}$ $(\mathrm{p}<0.0001)$ : no-MC 2.2\% ( $=9 ; 2.09 \times 1000$ PYs; Ref $)$, 1 MC 2.5\% $(\mathrm{n}=6 ; 2.37 \times 1000$ PYs; HR 1.12 [95\% CI
0.40-3.15]), 2 MC 19.7\% ( $\mathrm{n}=14 ; 19.62 \times 1000$ PYs; HR 9.12 [95\% CI 3.94-21.10]), 3 MC 27.3\% $(\mathrm{n}=6$; $39.13 \times 1000$ PYs; HR 18.35 [95\% CI 6.51-51.76]; Fig. 1c). In the adjusted Cox regression analysis (Fig. 4b, Models 1 to 3), the risk for coronary events, compared to no-MC, increased in subjects with $2 \mathrm{MC}$ and to a greater extent in those with $3 \mathrm{MC}$. In particular, in Model 3, HRs were: 1 MC 0.66 (95\% CI $0.23-$ 1.95), 2 MC 2.39 (95\% CI 0.85-6.69), 3 MC 5.26 (95\%

\footnotetext{
(See figure on next page.)

Fig. 1 Kaplan-Meier curves for all-cause death and cumulative incidences of major vascular and coronary events by MC. no-MC: dotted green line, 1 MC: dashed blue line, 2 MC: dashdotted violet line, 3 MC: solid red line. Percentages of events and Cox proportional unadjusted HRs (95\% $\mathrm{Cl}$ ) are shown for each MC group. a Survival from all-cause death: $\mathrm{K}-\mathrm{M}$ Logrank 187.35, $\mathrm{p}<0.0001$. Pairwise over strata logrank statistic provides the following results: 1 MC vs. no-MC, logrank 10.96 ( $p=0.0009$ ); 2 MC vs. no-MC, logrank 22.77 ( $p<0.0001$ ); furthermore, 3 MC vs. no-MC, logrank 223.18 , vs. 1 MC, logrank 78.94 and vs. 2 MC, logrank 30.62 ( $p<0.0001$, for all). b Cumulative incidence of major vascular events: K-M Logrank 127.61, $p<0.0001$. Pairwise over strata logrank statistic provides the following results: 1 MC vs. no-MC, logrank 3.71 ( $p=0.054$ ); 2 MC vs. no-MC, logrank 67.22 and vs. 1 MC, logrank 28.49 ( $p<0.0001$, for both); furthermore, 3 MC vs. no-MC, logrank 110.38, vs. 1 MC, logrank 57.81 ( $p<0.0001$, for both) and vs. 2 MC, logrank 4.15 ( $p=0.042)$. c Cumulative incidence of coronary events: K-M Logrank 81.47, $p<0.0001$. Pairwise over strata logrank statistic provides the following results: 2 MC vs. no-MC, logrank 40.60 and vs. 1 MC, logrank 26.17 ( $p<0.0001$, for both); 3 MC vs. no-MC, logrank 55.23 , vs. 1 MC, logrank 44.18 ( $p<0.0001$, for both)
} 
a

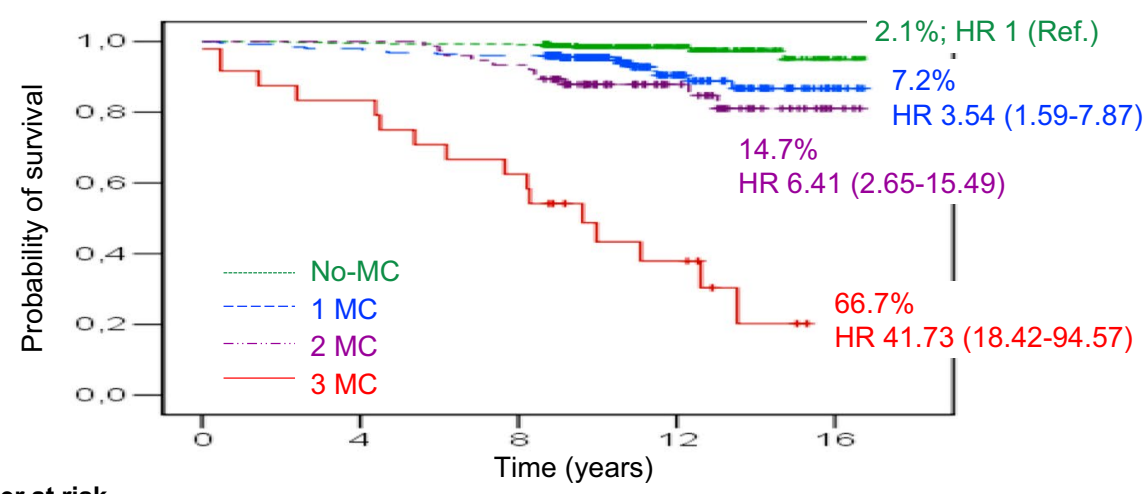

Number at risk

No-MC

$1 \mathrm{MC}$

$\begin{array}{cc}425 & 423 \\ 250 & 245 \\ 75 & 75 \\ 24 & 20 \\ 774 & 763\end{array}$

421

240

70

$3 \mathrm{MC}$

774

746

123

123
67

29

226

11
6
3
0
20

b

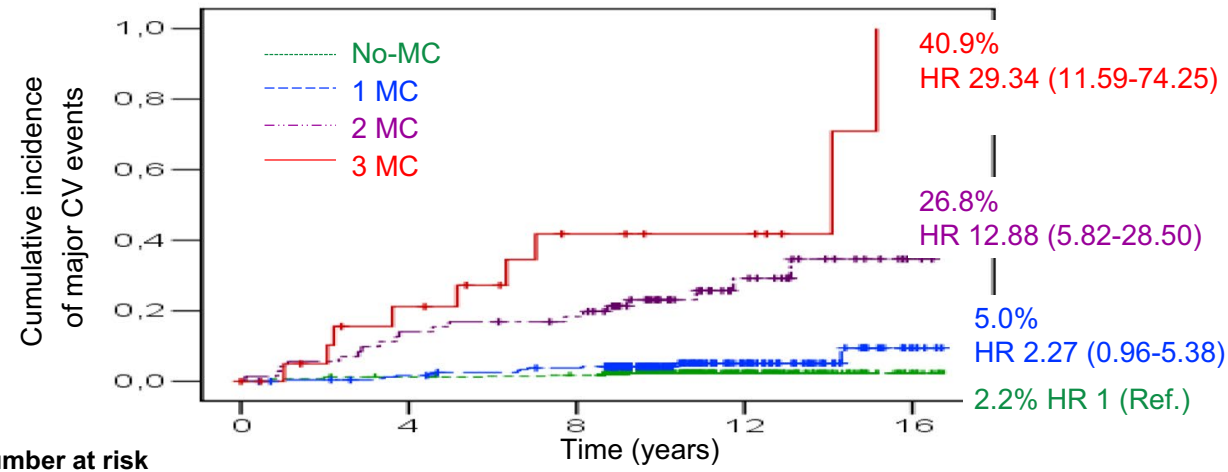

Number at risk

No-MC

$1 \mathrm{MC}$

$2 \mathrm{MC}$

$3 \mathrm{MC}$

$$
\begin{gathered}
402 \\
241 \\
71 \\
22 \\
736
\end{gathered}
$$

Total

$736 \quad 703$

395
233
61
14
703

391

225

56

7
679

$\begin{array}{cc}105 & 9 \\ 60 & 6 \\ 20 & 2 \\ 5 & 0 \\ \mathbf{1 9 0} & \mathbf{1 7}\end{array}$

c

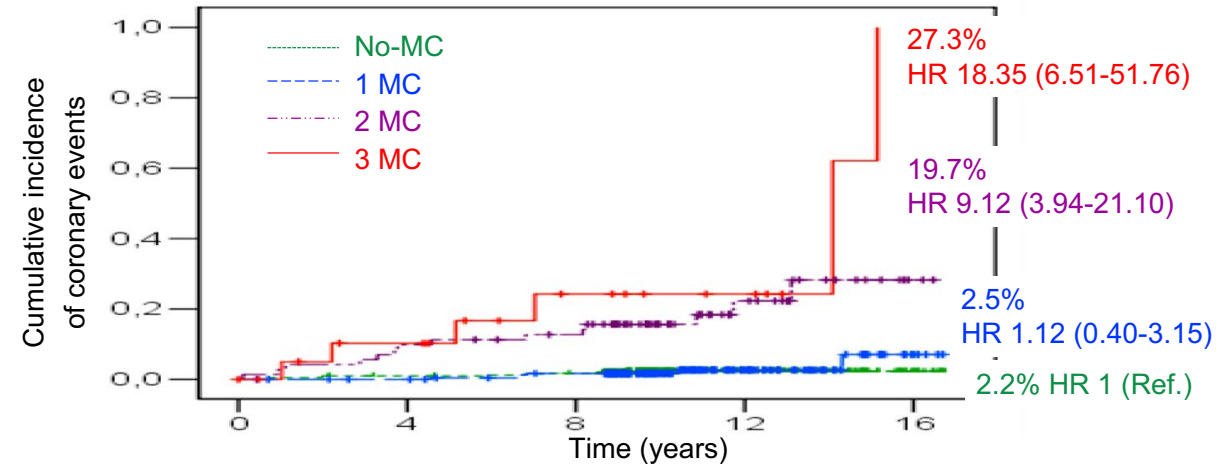

Number at risk

No-MC
$1 \mathrm{MC}$
$2 \mathrm{MC}$
$3 \mathrm{MC}$

402
241
71
22
736

396
237
64
16
713

391
228
59
9
687

105
60
20
5
190

9
6
2
0
17 

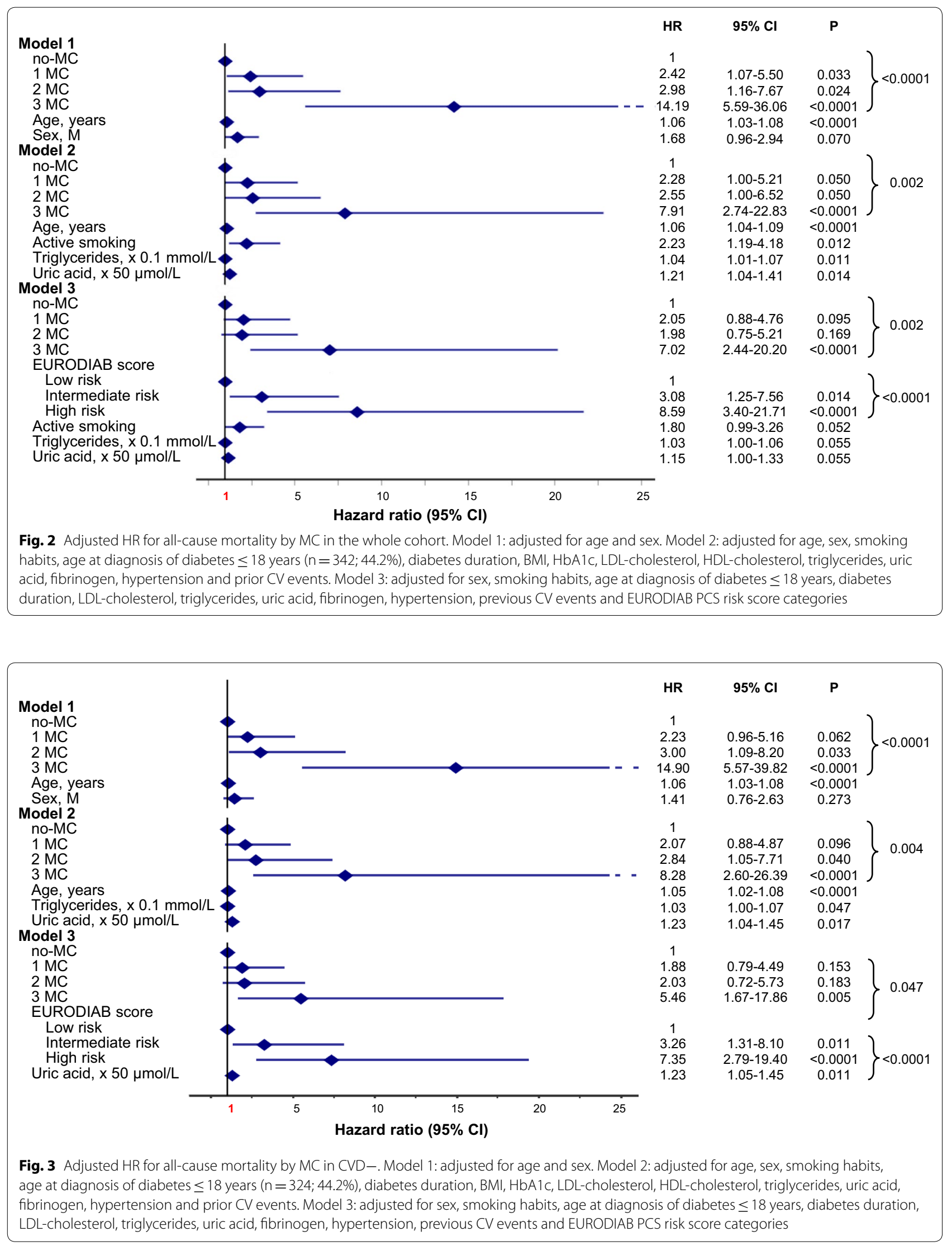


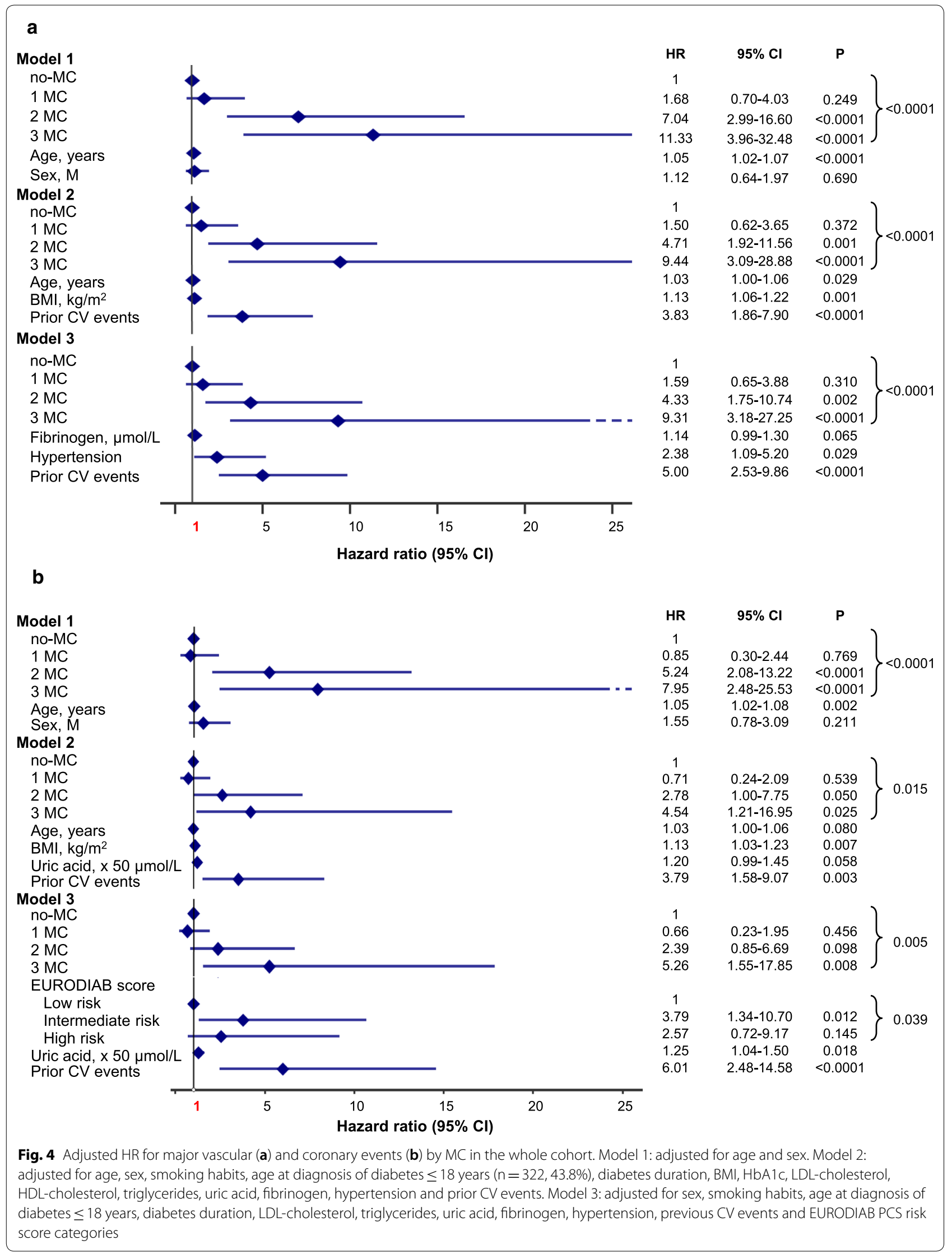




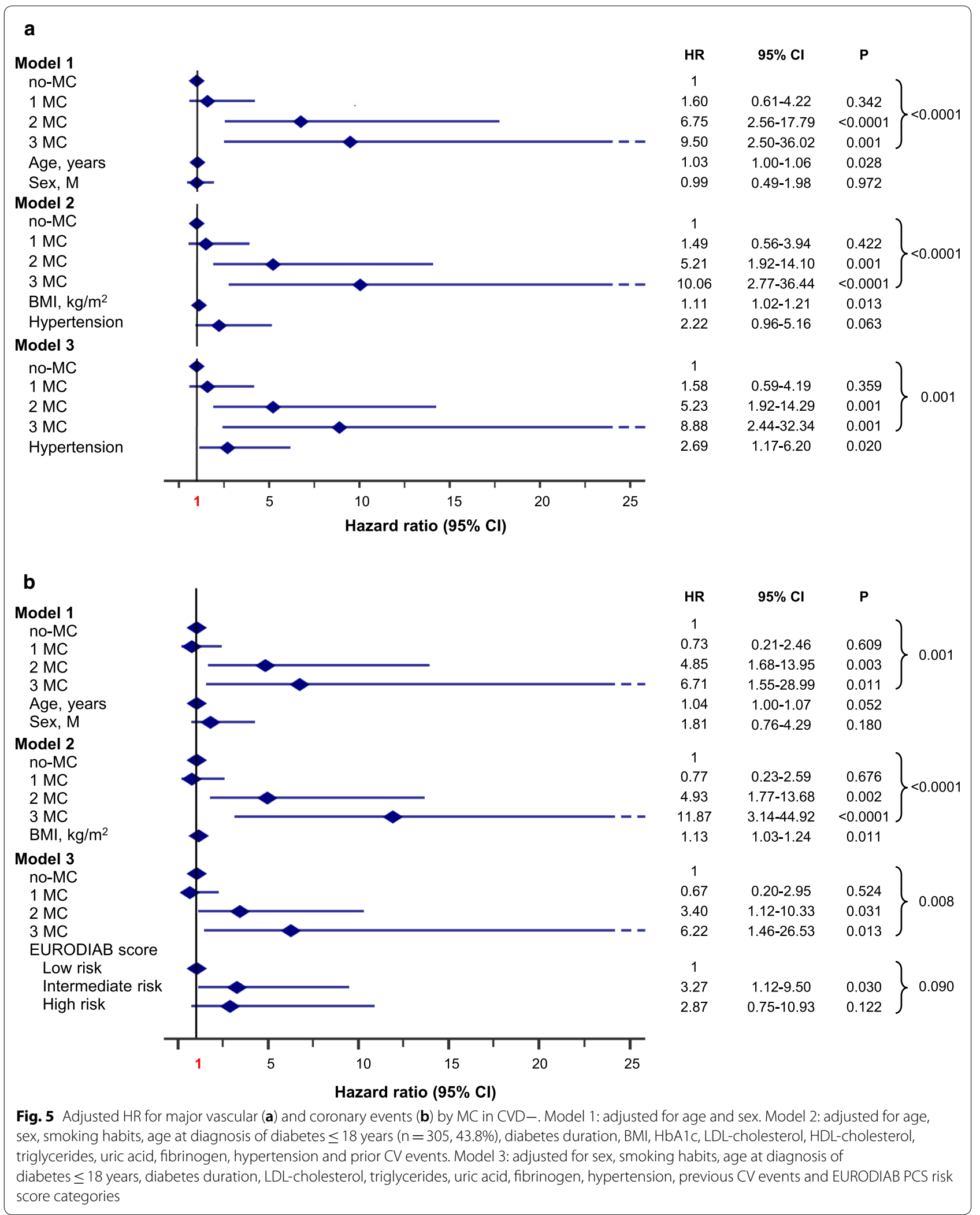


CI $1.55-17.85, \mathrm{p}=0.005)$ with independent effects for uric acid, EURODIAB PCS score and prior CV events.

\section{Microvascular complications and incidence of major cardiovascular and coronary events in CVD-}

Among CVD- $(n=697)$, incidence of major CV events $(\mathrm{n}=32,4.6 \%)$ increased with $\mathrm{MC}(\mathrm{p}<0.0001): 2.0 \%$ in no-MC $(\mathrm{n}=8 ; 1.88 \times 1000 \mathrm{PYs}), 3.9 \%$ in $1 \mathrm{MC}(\mathrm{n}=9$; $3.77 \times 1000 \mathrm{PYs}), 18.6 \%$ in $2 \mathrm{MC}(\mathrm{n}=11 ; 18.50 \times 1000$ PYs), and $26.7 \%$ in $3 \mathrm{MC}(\mathrm{n}=4 ; 32.50 \times 1000$ PYs; Additional file 1: Figure S3). The effect, though attenuated, remained significant after multiple adjustments up to Model 3, where the HR for $3 \mathrm{MC}$ resulted 8.88 (95\% CI 2.44-32.34, $\mathrm{p}=0.001$; Fig. 5a, Model 1 to 3), with independent effect for hypertension.

Consistently, incidence of coronary events $(n=23$, $3.3 \%)$ increased with $\mathrm{MC}(\mathrm{p}<0.0001): 2.0 \%$ in no-MC $(\mathrm{n}=8 ; 1.88 \times 1000 \mathrm{PYs}), 1.8 \%$ in $1 \mathrm{MC}(\mathrm{n}=4 ; 1.66 \times 1000$ PYs), $13.6 \%$ in $2 \mathrm{MC}(\mathrm{n}=8 ; 13.24 \times 1000 \mathrm{PYs})$, and $20.0 \%$ in $3 \mathrm{MC}(\mathrm{n}=3 ; 23.47 \times 1000 \mathrm{PYs}$; Additional file 1 : Figure S4). Once again, the effect remained significant even after multiple adjustments (Fig. 5b, Model 1 to 3 ).

\section{Retinopathy, mortality and other outcomes}

Diabetic retinopathy was the most frequent $\mathrm{MC}(\mathrm{n}=322$; 41.6\%); 82 individuals had nephropathy (10.6\%) and 68 neuropathy $(8.8 \%)$. The prevalence of advanced retinopathy increased from individuals with 1 to those with $3 \mathrm{MC}$ ( $\mathrm{p}<0.0001$; Additional file 1: Figure S1).

Mortality was $2.7 \%(n=12 ; 2.41 \times 1000$ PYs; Ref $)$ in 452 subjects without DR (DR-) and increased to $13.0 \%$ $(\mathrm{n}=42 ; 12.36 \times 1000$ PYs, HR 5.18 [95\% CI 2.72-9.83]) in the remaining $322 \mathrm{DR}+$ individuals $(\mathrm{p}<0.0001$; Additional file 1: Figure S5A). Adjustment for nephropathy and neuropathy did not reduce the risk for mortality associated with DR+ (HR 2.71 [95\% CI 1.355.43]; $\mathrm{p}=0.005$ ) nor it did adjustment for other potential confounders (Additional file 1: Table S3, Models 1 to 3). In Model 3, death HRs were 2.07 (95\% CI 1.02-4.19; $\mathrm{p}=0.044)$ for $\mathrm{DR}+$, and 3.02 (95\% CI 1.24-7.36) and 9.65 (95\% CI 4.02-23.16), respectively, for intermediateand high-risk EURODIAB PCS score $(\mathrm{p}<0.0001)$, with an independent role for nephropathy, smoking, and uric acid. Mortality occurred in $9.4 \%$ among subjects with non-advanced DR $(n=19$ out of $202 ; 8.91 \times 1000$ PYs; HR 3.74 [95\% CI 1.81-7.70]) versus $19.2 \%$ in those with advanced DR ( $\mathrm{n}=23$ out of $120 ; 18.16 \times 1000$ PYs; HR 7.60 [95\% CI 3.78-15.28]; $\mathrm{p}<0.0001$; Additional file 1: Figure S5B). HRs remained significant with no difference between non-advanced and advanced DR (2.71 [95\% CI 1.30-5.69] and 2.69 [95\% CI 1.20-6.05]; $\mathrm{p}=0.008$ and $\mathrm{p}=0.017$, respectively) after adjustment for nephropathy and neuropathy. Mortality HRs remained significant for non-advanced DR after adjustment for confounders, with an independent effects for active smoking, uric acid, and EURODIAB PCS score (data not shown).

Cumulative incidence of major CV events was $2.1 \%$ ( $\mathrm{n}=9 ; 1.97 \times 1000$ PYs; Ref) among $426 \mathrm{DR}-$ and $12.9 \%$ $(\mathrm{n}=40 ; 13.05 \times 1000$ PYs; HR 6.59 [95\% CI 3.20-13.59]) in $310 \mathrm{DR}+(\mathrm{p}<0.0001$; Additional file 1: Figure S6A). In this case too, the risk remained significantly higher after adjustment for nephropathy and neuropathy, and several other confounders (Additional file 1: Table S4, Models 1 to 3). In Model 3, HRs for CV events were 2.76 (95\% CI 1.25-6.07; $\mathrm{p}=0.012$ ) for DR+, and 2.31 (95\% CI $0.98-$ 5.41) and 3.71 (95\% CI 1.47-9.37) for intermediate- and high-risk EURODIAB PCS score, respectively $(\mathrm{p}=0.021)$, with an independent role for nephropathy and prior $\mathrm{CV}$ events. Moreover, the event rate was lower for nonadvanced as compared to advanced DR ( $\mathrm{p}<0.0001$; Additional file 1: Figure S6B), even after adjustment for the other MC and confounders (data not shown). A similar trend was observed as far incidence of coronary events (Additional file 1: Figures S7A, B), although in Model 2 and 3 DR did not enter as a covariate of coronary events, while independent roles were played by nephropathy, prior CV disease, uric acid and EURODIAB score (Additional file 1: Table S5, Models 1 to 3).

For each outcome, in all regression models no interaction ( $p \geq 0.368$ for all, data not shown) has been observed between nephropathy or neuropathy with the presence of retinopathy even if, as anticipated by the low number of individuals with nephropathy or neuropathy free from retinopathy, confidence intervals should, in many cases, interpreted with caution.

\section{Discussion}

We found that in a cohort of 774 subjects with type 1 diabetes, over a more than 10 year follow-up the presence of microvascular disease at study entry was an independent determinant of all-cause mortality and cardiovascular events. Moreover, we found that number and severity of microvascular complications were associated with an increase in the rate of all-cause death, first major cardiovascular and first coronary event. The presence of one or more MC was also associated with worse profile of conventional and non conventional $\mathrm{CV}$ risk factors, including a contemporary specific vascular risk score. Yet, even accounting for these variables, $\mathrm{MC}$ remained an independent predictor of major outcomes, including allcause mortality and CV events. These observations were fully confirmed in the 733 individuals free of established CV disease at baseline. Finally, retinopathy, the most frequent $\mathrm{MC}$ in our cohort, conferred by itself a higher risk of mortality and $\mathrm{CV}$ outcomes irrespective of $\mathrm{CV}$ risk profile and of the effect of nephropathy and neuropathy. 
The pathophysiologic processes leading to $\mathrm{CV}$ disease in type 1 diabetes are complex and largely unidentified. Microvascular beds are the sites where the earliest consequences of inflammatory processes occur, leading to endothelial and smooth muscle cell dysfunction, and altered structure and composition of the extracellular matrix. These modifications are recognized as risk factors for the development of atherosclerotic disease in large arteries as well [30]. Also, a rule in diabetic vascular complications has been suggested for advanced glycation end-products assessed by skin autofluorescence [31, 32], HDL dysfunction [33], and altered regulation of extracellular matrix remodeling [34], both in type 1 and type 2 diabetes. Among microvascular beds, the eye and the kidney are vulnerable target end-organs where functional and structural microvascular alterations anticipate and predict incident CV events [30].

In line with all this, recent cross-sectional studies reported an association between microvascular complications, mainly diabetic retinopathy, and subclinical atherosclerosis in subjects with type 1 diabetes. In a cohort of subjects with type 1 diabetes without previous CV disease or DKD, Carbonell et al. found an independent association between PDR and higher atherosclerotic burden in the carotid arteries [35]. Furthermore, in subjects with long-lasting diabetes ( $\geq 50$ years) Lovshin et al. found that retinopathy and large-nerve fiber neuropathy were associated with presence of higher coronary artery calcification volume score [36].

These observations suggest that involvement of microvascular beds may reflect underlying, as jet unidentified, pathways linked to vascular disease.

An association between microvascular complications and risk of $\mathrm{CV}$ disease has been recently reported in type 2 diabetes. A cohort of 49,027 individuals with type 2 diabetes and no history of $\mathrm{CV}$ disease at baseline was studied by Brownrigg et al. over a median follow-up of 5.5 years [37]. In this study, the investigators found a dose-dependent relationship between the number of microvascular complications, the overall risk of experiencing a CV event, as well as risk of other endpoints such as all-cause mortality, $\mathrm{CV}$ death and hospitalization for heart failure. The same study found that isolated retinopathy, peripheral neuropathy or nephropathy, independent of conventional risk factors, conferred risk of $\mathrm{CV}$ events similar to the one associated with blood pressure, $\mathrm{HbA}_{1 \mathrm{c}}$ and LDL-cholesterol. Furthermore, no deviation from linearity was found in subgroups stratified by varying degrees of risk factors control [37].

On the contrary, robust data assessing the effects of microvascular complications on all-cause mortality and CV risk are still lacking in type 1 diabetes [38]. Previous reports have shown an increase in CV risk and mortality in subjects with type 1 diabetes with diabetic kidney disease $[9,12,39-42]$ and peripheral [43] or autonomic neuropathy [43-45]. A recent report from FinnDiane showed that in subjects with long-standing type 1 diabetes severe $\mathrm{DR}$, even without DKD, increases the risk of CV disease, in particular for peripheral artery disease, independently of common CV risk factors [46].

We now provide evidence that incidence of allcause death increases in individuals with type 1 diabetes with one (6.76/1000 PYs), two (12.93/1000 PYs) or three (79.77/1000 PYs) as compared to subjects free of microvessel damage (1.92/1000 PYs). Adjustment for potential confounders attenuated HR for primary outcome across all three groups, but the microvascular burden remained an independent predictor in particular for those individuals with $3 \mathrm{MC}$. This was also true among those subjects without prior $\mathrm{CV}$ events even after correction for the EURODIAB PCS score, a powerful integrated predictor of major outcomes including all-cause mortality. The association is similar, if not stronger, between cumulative burden of $\mathrm{MC}$ and major vascular events. Unadjusted rates were $2.09,4.79,27.56$, and $63.82 / 1000$ PYs for individuals with no-MC and for those with one, two, or three MC, respectively. Again HRs for major vascular outcomes were attenuated across the three groups after adjustment for confounders. A similar pattern was observed for coronary events.

The attenuation of the HRs for each outcome after multiple adjustments confirms the role of conventional $\mathrm{CV}$ risk factors. Yet, the persistence of the predictive effect of MC, even after full adjustment (i.e. Model 3), supports the negative impact of existing microvascular complications, which exert an effect similar to the one of active smoking on all-cause mortality (Fig. 2) [47]. Also, the coexistence of two microvascular complications is a stronger predictor for major CV events than hypertension (Fig. 4a) and three $\mathrm{MC}$ are associated with coronary events to a greater extent than the EURODIAB PCS score (Fig. 4b).

We found no independent association of baseline $\mathrm{HbA}_{1 \mathrm{c}}$ with mortality, major vascular outcomes or coronary events. Besides the much debated effect of glycaemic control on these outcomes, the present finding should be interpreted with caution as a single baseline $\mathrm{HbA}_{1 \mathrm{c}}$ value is unlikely to capture and predict cumulative exposure to hyperglycemia. Also, one could argue that glycaemic control is well incorporated in the degree of the microvascular bed involvement given the well known relationship between glucose control and development of microvessel damage.

Due to the size of our study population, we could not analyze data according to individual effect of retinopathy, nephropathy or peripheral neuropathy. However, we 
found that retinopathy, the most common microvascular complication in our cohort, conferred per se an increased risk for all-cause mortality and vascular outcomes, an association that remained valid after adjustment for concomitant nephropathy and peripheral neuropathy as well as adjustment for other confounders.

This particular finding is at variance with the results very recently published by Bjerg et al. [15]. In a larger population of 3828 subjects with type 1 diabetes over a 26,665 PYs follow-up they found that neuropathy and diabetic kidney disease were strong and independent risk factors of mortality with no evidence for an association with retinopathy. Furthermore, on the contrary of the present study, authors found no evidence that multiple complications could increase the risk conferred by each complication separately [15]. A number of reasons could account for these discrepancies, including difference in population size. Since diabetic retinopathy was by far the most common microvascular condition in a smaller population with a longer follow-up, this may have greater chance to show a significant association. Nonetheless, the relative role of microvascular complications on mortality and cardiovascular risk in type 1 diabetes may require more attention. Systematic reviews have recently examined the associations between diabetic retinopathy and common macrovascular complications $[48,49]$. The analysis by Pearce et al. included both type 1 and type 2 diabetes and showed that DR was consistently associated with other complications of diabetes. In keeping with our own results, the meta-analysis also reported an association between severity of DR and CV risk. In particular, DR was a strong predictor of stroke, major CV events and peripheral artery disease [48]. In the meta-analysis by Guo et al. DR was associated with increased risk of CV disease (relative risk 2.42 [95\% CI 1.77-3.31]); this risk was stronger in type 1 diabetes (relative risk 3.59 [95\% CI 1.79-7.20]) [49]. A recent report from FinnDiane found that severe DR, even in the absence of concomitant DKD, increases cardiovascular risk, in particular the risk of peripheral artery disease, independently of common CV risk factors [46]. Moreover, the Joslin Medalist Study showed that the CV risk was greater in subjects with severe DR on top of DKD than in those with DKD alone [14]. In summary, besides the discrepancy with respect to the specific effect of DR, our study and the one by Bjerg et al. [15] support the concept that microvascular complications can contribute in increasing the risk of all-cause and cardiovascular mortality in subjects with type 1 diabetes.

There are a number of limitations of the present study. First, the number of events (54 deaths and 49 major CV events) was limited suggesting some caution should be used in extrapolating from multivariate analysis and its clinical implications. Second, we have no information on the course of microvascular diseases over time; this precluded us to assess the effects of progression or regression of the microvascular involvement on relevant outcomes. For instance, while progression of diabetic nephropathy confers an increased risk for cardiovascular disease and premature death, it has been recently reported that, in type 1 diabetes, regression of albuminuria reduces the risk of cardiovascular events and all-cause and cardiovascular mortality to the same level as for those who did not progress [50]. A similar limitation of the study is the lack of data on time-varying exposure to glycated haemoglobin levels and conventional cardiovascular risk factors during follow-up. Third, data on autonomic neuropathy were not available at baseline. In the Diabetes Control and Complications Trial/Epidemiology of Diabetes Intervention and Complications (DCCT/EDIC) cohort, individuals with cardiovascular autonomic neuropathy at study closeout had higher long-term risk of CV events [45]. A further limitation of our study is that we could not retrieve reliable data about cause of death, including CV death, from the Regional Registry, somewhat limiting the detail of study results. Finally, the results of this study should be interpreted taking into consideration the characteristics of our cohort, with retinopathy being by far the most frequent microvascular complication. Thus, in addition to the co-existence of microvascular complications, also the severity of retinopathy (as an alternative way to assess the degree of microvascular burden) has been associated with each study outcome.

The study, however, also have some strengths, such as the single-centre nature of the study and the relatively long follow-up. Moreover, registry linkage with individuals' unique personal identification codes provides survival information for virtually all participants and 95\% coverage for major vascular events. Of relevance, details on retinopathy classification was available for all individuals allowing meaningful sensitivity analyses for advanced and early stages of the condition.

\section{Conclusions}

In summary, our data corroborate initial information and more recent publications [15] that the presence and severity of microvascular complications contribute in an independent manner to increase the risk of all-cause mortality and cardiovascular outcomes in individuals with type 1 diabetes. As the assessment of microvascular disease is an essential component of routine clinical care, these findings show how screening and staging for microvascular complications can offer a convenient and unexpensive tool for improving risk prediction in subjects with type 1 diabetes. 


\section{Supplementary information}

Supplementary information accompanies this paper at https://doi. org/10.1186/s12933-019-0961-7.

Additional file 1: Table S1. International Classification of Diseases (ICD-9) system codes collected during follow-up. Table S2. Baseline clinical characteristics of individuals with retinopathy, nephropathy and neuropathy compared with those with no MC. Table S3. Survival analysis by Cox proportional hazards regression according to presence of any degree of retinopathy at baseline. Table S4. Incidence analysis of major CV events by Cox proportional hazards regression according to presence of any degree of retinopathy at baseline. Table S5. Incidence analysis of coronary events by Cox proportional hazards regression according to presence of any degree of retinopathy at baseline. Figure S1. Distribution of microvascular diseases in the 774 participants with type 1 diabetes. Figure S2. Kaplan-Meier curves showing survival from all-cause death by MC groups in individuals without prior CV events. Figure S3. Kaplan-Meier curves showing cumulative incidence of major vascular events by MC groups in individuals without prior CV events. Figure S4. Kaplan-Meier curves showing cumulative incidence of coronary events in by $\mathrm{MC}$ groups in individuals without prior CV events. Figure S5. Kaplan-Meier curves showing survival from all-cause death in participants with retinopathy vs. those without DR. Figure S6. Kaplan-Meier curves showing cumulative incidence of major CV events in participants with retinopathy vs. those without DR. Figure S7. Kaplan-Meier curves showing cumulative incidence of coronary events in participants with retinopathy vs. those without DR.

\section{Abbreviations}

ACR: albumin-to-creatinine ratio; ALT: alanine aminotransferase; AST: aspartate aminotransferase; BMl: body mass index; BP: blood pressure; CHD: coronary artery disease; Cl: confidence intervals; CKD-EPI: chronic kidney disease epidemiology collaboration equation; CV: cardiovascular; CVD: cardiovascular disease; DD: diabetes duration; DKD: diabetic kidney disease; DR: diabetic retinopathy; EURODIAB PCS: EURODIAB Prospective Complications Study; eGFR: estimated glomerular filtration rate; GGT: gamma-glutamyltransferase; HR: hazard ratio; HS: high score; IS: intermediate score; K-M: Kaplan-Meier; LE: life expectancy; LS: low score; MC: microvascular complication; PDR: proliferative diabetic retinopathy; PYS: person-years; RAS: renin angiotensin system; WHR: waist-to-hip ratio.

\section{Acknowledgements}

We are indebted to all patients attending the Outpatients Diabetic Clinic and to the staff of the "Renzo Navalesi" Diabetes Centre in Pisa, in particular to the Clinical Lab and the Medical Records Unit.

\section{Authors' contributions}

MG, GP and SDP designed the study, drafted the manuscript and approved its final version; MG, GD, RM, GP, and DL contributed to the study design and to analysis and interpretation of data, revised the article's intellectual content and approved its final version; MG, EG, PF, RG, MA and FC acquired data, revised the article's intellectual content and approved its final version. MG, GP and SDP take full responsibility for the integrity of this work as a whole, including the study design, access to data, and the decision to submit and publish the manuscript. All authors read and approved the final manuscript.

\section{Funding}

This work was supported by a grant from the Regione Toscana, Italy, Resolution 1157 (December 19, 2011), ID Number D55E11002680005. The funder had no role in the original study design, data collection, data analysis, data interpretation, writing of the manuscript, or decision to submit the report for publication. All authors had full access to all the data of the study, and the corresponding author had final responsibility for the decision to submit for publication.

\section{Availability of data and materials}

Data collected for this study can be shared and made available upon reasonable request to the corresponding author and subject to an approved proposal and data access agreement.
Ethics approval and consent to participate

The local ethics committee approved the study protocol, and the study was carried out in accordance with Declaration of Helsinki. Each participant signed a written informed consent.

\section{Consent for publication}

Not applicable.

\section{Competing interests}

MG has received travel grants from AstraZeneca and Novo Nordisk. MA has received speaker/advisory honoraria from Abbott Laboratories. FC has received travel grants from Novo Nordisk. GD reports past participation in advisory boards from AstraZeneca, Eli Lilly and Co and Novartis Pharmaceuticals. SDP reports past participation in advisory boards and/or receiving research grants from Abbott Laboratories, AstraZeneca, Boehringer Ingelheim, Eli Lilly and Co, Merck Sharp \& Dohme, Novartis Pharmaceuticals, Novo Nordisk, Sanofi, Servier, and Takeda Pharmaceuticals. GP has received travel grants and speaker/advisory honoraria from AstraZeneca, Boehringer Ingelheim, Eli Lilly and Co, Merck Sharp \& Dohme, Mundipharma Pharmaceuticals, Novo Nordisk, and Takeda Pharmaceuticals. All other authors (EG, RG, DL, MR, and PF) declare that they have no competing interests.

\section{Author details}

1 Section of Diabetes and Metabolic Disease, Department of Clinical and Experimental Medicine, University of Pisa and Azienda Ospedaliero-Universitaria Pisana, Via Paradisa, 2, 56124 Pisa, Italy. ${ }^{2}$ Epidemiology Unit, Regional Health Agency (ARS) of Tuscany, Florence, Italy.

Received: 31 July 2019 Accepted: 1 November 2019 Published online: 16 November 2019

\section{References}

1. Rawshani A, Rawshani A, Franzén S, Eliasson B, Svensson AM, Miftaraj M, et al. Mortality and cardiovascular disease in type 1 and type 2 diabetes. N Engl J Med. 2017;376(15):1407-18.

2. Diabetes Control and Complications Trial (DCCT)/Epidemiology of Diabetes Interventions and Complications (EDIC) Study Research Group. Mortality in type 1 diabetes in the DCCT/EDIC versus the general population. Diabetes Care. 2016;39(8):1378-83.

3. Secrest AM, Becker DJ, Kelsey SF, LaPorte RE, Orchard TJ. All-cause mortality trends in a large population-based cohort with long-standing childhood-onset type 1 diabetes: the Allegheny County type 1 diabetes registry. Diabetes Care. 2010;33(12):2573-9.

4. Soedamah-Muthu SS, Fuller JH, Mulnier HE, Raleigh VS, Lawrenson RA, Colhoun HM. All-cause mortality rates in patients with type 1 diabetes mellitus compared with a non-diabetic population from the UK general practice research database, 1992-1999. Diabetologia. 2006;49(4):660-6.

5. Jørgensen ME, Almdal TP, Carstensen B. Time trends in mortality rates in type 1 diabetes from 2002 to 2011. Diabetologia. 2013;56(11):2401-4.

6. Petrie D, Lung TW, Rawshani A, Palmer AJ, Svensson AM, Eliasson B, et al. Recent trends in life expectancy for people with type 1 diabetes in Sweden. Diabetologia. 2016;59(6):1167-76.

7. Huo L, Harding JL, Peeters A, Shaw JE, Magliano DJ. Life expectancy of type 1 diabetic patients during 1997-2010: a national Australian registrybased cohort study. Diabetologia. 2016:59(6):1177-85.

8. Rawshani A, Rawshani A, Franzén S, Eliasson B, Svensson AM, Miftaraj $M$, et al. Range of risk factor levels: control, mortality, and cardiovascular outcomes in type 1 diabetes mellitus. Circulation. 2017;135(16):1522-31.

9. Lind M, Svensson AM, Kosiborod M, Gudbjörnsdottir S, Pivodic A, Wedel $\mathrm{H}$, et al. Glycemic control and excess mortality in type 1 diabetes. N Engl J Med. 2014;371(21):1972-82.

10. Soedamah-Muthu SS, Vergouwe Y, Costacou T, Miller RG, Zgibor J, Chaturvedi $\mathrm{N}$, et al. Predicting major outcomes in type 1 diabetes: a model development and validation study. Diabetologia. 2014;57(11):2304-14.

11. Vistisen D, Andersen GS, Hansen CS, Hulman A, Henriksen JE, BechNielsen $\mathrm{H}$, et al. Prediction of first cardiovascular disease event in type 1 diabetes mellitus: the steno type 1 risk engine. Circulation. 2016;133(11):1058-66. 
12. Groop PH, Thomas MC, Moran JL, Wadèn J, Thorn LM, Mäkinen VP, et al. FinnDiane Study Group. The presence and severity of chronic kidney disease predicts all-cause mortality in type 1 diabetes. Diabetes. 2009;58(7):1651-8.

13. van Hecke MV, Dekker JM, Stehouwer CD, Polak BC, Fuller JH, Sjolie AK, et al. EURODIAB prospective complications study. Diabetic retinopathy is associated with mortality and cardiovascular disease incidence: the EURODIAB prospective complications study. Diabetes Care. 2005;28(6):1383-9.

14. Gordin D, Harjutsalo V, Tinsley L, Fickweiler W, Sun JK, Forsblom C, et al. Differential association of microvascular attributions with cardiovascular disease in patients with long duration of type 1 diabetes. Diabetes Care. 2018;41(4):815-22.

15. Bjerg L, Hulman A, Carstensen B, Charles M, Witte DR, Jørgensen ME. Effect of duration and burden of microvascular complications on mortality rate in type 1 diabetes: an observational clinical cohort study. Diabetologia. 2019;62(4):633-43.

16. Pease A, Earnest A, Ranasinha S, Nanayakkara N, Liew D, Wischer N, et al. Burden of cardiovascular risk factors and disease among patients with type 1 diabetes: results of the Australian National Diabetes Audit (ANDA). Cardiovasc Diabetol. 2018;17(1):77

17. Heller T, Kloos C, Lehmann T, Schiel R, Lorkowski S, Wolf G, et al. Mortality and its causes in a german cohort with diabetes mellitus type 1 after 20 years of follow-up: the JEVIN Trial. Exp Clin Endocrinol Diabetes. 2018;126(6):387-93.

18. Penno G, Russo E, Garofolo M, Daniele G, Lucchesi D, Giusti L, et al. Evidence for two distinct phenotypes of chronic kidney disease in individuals with type 1 diabetes mellitus. Diabetologia. 2017;60(6):1102-13.

19. Stephenson J, Fuller JH. on behalf of the EURODIAB IDDM Complications Study Group. Microvascular and acute complications in IDDM patients: the EURODIAB IDDM Complications Study. Diabetologia. 1994;37(3):278-85.

20. International Federation of Clinical Chemistry and Laboratory Medicine, IFCC Scientific Division, Mosca A, Goodall I, Hoshino T, Jeppsson JO, John WG, Little RR, et al. Global standardization of glycated hemoglobin measurement: the position of the IFCC Working Group. Clin Chem Lab Med. 2007;45(8):1077-80.

21. Friedewald WT, Levy RI, Fredrickson DS. Estimation of the concentration of low-density lipoprotein cholesterol in plasma, without use of the preparative ultracentrifuge. Clin Chem. 1972;18:499-502.

22. Levey AS, Stevens LA, Schmid CH, Zhang YL, Castro AF, Feldman HI, et al. CKD-EPI (Chronic Kidney Disease Epidemiology Collaboration). A new equation to estimate glomerular filtration rate. Ann Intern Med. 2009;150(9):604-12.

23. Levey AS, Cattran D, Friedman A, Miller WG, Sedor J, Tuttle K, et al. Proteinuria as a surrogate outcome in CKD: report of a scientific workshop sponsored by the National Kidney Foundation and the US Food and Drug Administration. Am J Kidney Dis. 2009;54(2):205-26.

24. Kumar R. Kidney Disease: improving Global Outcomes (KDIGO) CKD Work Group. KDIGO clinical practice guideline for the evaluation and management of chronic kidney disease. Kidney Int. 2013;3:1-150.

25. Aldington SJ, Kohner EM, Meuer S, Klein R, Sjølie AK. Methodology for retinal photography and assessment of diabetic retinopathy: the EURODIAB IDDM complications study. Diabetologia. 1995;38(4):437-44.

26. Wilkinson CP, Ferris FL, Klein RE, Lee PP, Agardh CD, Davis M, et al. Global Diabetic Retinopathy Project Group. Proposed international clinical diabetic retinopathy and diabetic macular edema disease severity scales. Ophthalmology. 2003;110(9):1677-82.

27. Herman WH, Pop-Busui R, Braffett BH, Martin CL, Cleary PA, Albers JW, et al. DCCT/EDIC Research Group. Use of the Michigan neuropathy screening instrument as a measure of distal symmetrical peripheral neuropathy in Type 1 diabetes: results from the diabetes control and complications trial/epidemiology of diabetes interventions and complications. Diabet Med. 2012;29(7):937-44.

28. Maffei L, Premrou V, Roldan P, Copetti M, Pellegrini F, Rossi MC, et al. Vibration perception threshold in the screening of sensorimotor distal symmetric polyneuropathy: the need of more accurate age-specific reference values. J Diabetes Sci Technol. 2014;8(3):621-2.

29. Prineas RJ, Crow RS, Blackburn H. The minnesota code manual of electrocardiographic findings. Boston: JohnWright-PSG; 1982.
30. Lockhart CJ, McCann AJ, Pinnock RA, Hamilton PK, Harbinson MT, McVeigh GE. Multimodal functional and anatomic imaging identifies preclinical microvascular abnormalities in type 1 diabetes mellitus. Am J Physiol Heart Circ Physiol. 2014;307(12):H1729-36.

31. Blanc-Bisson C, Velayoudom-Cephise FL, Cougnard-Gregoire A, Helmer C, Rajaobelina K, Delcourt C, et al. Skin autofluorescence predicts major adverse cardiovascular events in patients with type 1 diabetes: a 7-year follow-up study. Cardiovasc Diabetol. 2018;17(1):82.

32. Yozgatli K, Lefrandt JD, Noordzij MJ, Oomen PHN, Brouwer T, Jager J, et al. Accumulation of advanced glycation end products is associated with macrovascular events and glycaemic control with microvascular complications in type 2 diabetes mellitus. Diabet Med. 2018;35(9):1242-8.

33. Heier M, Borja MS, Brunborg C, Seljeflot I, Margeirsdottir HD, Hanssen KF, et al. Reduced HDL function in children and young adults with type 1 diabetes. Cardiovasc Diabetol. 2017;16(1):85

34. Peeters SA, Engelen L, Buijs J, Jorsal A, Parving HH, Tarnow L, et al. Plasma matrix metalloproteinases are associated with incident cardiovascular disease and all-cause mortality in patients with type 1 diabetes: a 12-year follow-up study. Cardiovasc Diabetol. 2017;16(1):55.

35. Carbonell M, Castelblanco E, Valldeperas X, Betriu A, Traveset A, GranadoCasas $M$, et al. Diabetic retinopathy is associated with the presence and burden of subclinical carotid atherosclerosis in type 1 diabetes. Cardiovasc Diabetol. 2018;17(1):66.

36. Lovshin JA, Bjornstad P, Lovblom LE, Bai JW, Lytvyn Y, Boulet G, et al. Atherosclerosis and microvascular complications: results from the canadian study of longevity in type 1 diabetes. Diabetes Care. 2018;41(12):2570-8.

37. Brownrigg JR, Hughes CO, Burleigh D, Karthikesalingam A, Patterson $\mathrm{BO}$, Holt PJ, et al. Microvascular disease and risk of cardiovascular events among individuals with type 2 diabetes: a population-level cohort study. Lancet Diabetes Endocrinol. 2016;4(7):588-97.

38. Sauder KA, Stafford JM, Mayer-Davis EJ, Jensen ET, Saydah S, Mottl A, et al. SEARCH for Diabetes in Youth Study Group. Co-occurrence of early diabetes-related complications in adolescents and young adults with type 1 diabetes: an observational cohort study. Lancet Child Adolesc Health. 2019;3(1):35-43.

39. Thorn LM, Gordin D, Harjutsalo V, Hägg S, Masar R, Saraheimo M, et al. FinnDiane Study Group. The presence and consequence of nonalbuminuric chronic kidney disease in patients with type 1 diabetes. Diabetes Care. 2015;38(11):2128-33.

40. Garofolo M, Russo E, Miccoli R, Lucchesi D, Giusti L, Sancho-Bornez V, et al. Albuminuric and non-albuminuric chronic kidney disease in type 1 diabetes: association with major vascular outcomes risk and all-cause mortality. J Diabetes Compl. 2018;32(6):550-7.

41. Harjutsalo V, Thomas MC, Forsblom C, Groop PH, FinnDiane Study Group. Risk of coronary artery disease and stroke according to sex and presence of diabetic nephropathy in type 1 diabetes. Diabetes Obes Metab. 2018;20(12):2759-67

42. Marcovecchio ML, Chiesa ST, Armitage J, Daneman D, Donaghue KC, Jones TW, et al. Adolescent Type 1 Diabetes Cardio-Renal Intervention Trial (AdDIT) Study Group. Renal and cardiovascular risk according to tertiles of urinary albumin-to-creatinine ratio: the adolescent type 1 diabetes cardio-renal intervention trial (AdDIT). Diabetes Care. 2018;41(9):1963-9.

43. Soedamah-Muthu SS, Chaturvedi N, Witte DR, Stevens LK, Porta M, Fuller JH. EURODIAB Prospective Complications Study Group. Relationship between risk factors and mortality in type 1 diabetic patients in Europe: the EURODIAB Prospective Complications Study (PCS). Diabetes Care. 2008;31(7):1360-6.

44. Mogensen UM, Jensen T, Køber L, Kelbæk H, Mathiesen AS, Dixen U, et al. Cardiovascular autonomic neuropathy and subclinical cardiovascular disease in normoalbuminuric type 1 diabetic patients. Diabetes. 2012;61(7):1822-30

45. Pop-Busui R, Braffett BH, Zinman B, Martin C, White NH, Herman WH, et al. DCCT/EDIC Research Group. cardiovascular autonomic neuropathy and cardiovascular outcomes in the diabetes control and complications trial/ epidemiology of diabetes interventions and complications (DCCT/EDIC) study. Diabetes Care. 2017:40(1):94-100.

46. Pongrac Barlovic D, Harjutsalo V, Gordin D, Kallio M, Forsblom C, King G, et al. FinnDiane Study Group. The association of severe diabetic retinopathy with cardiovascular outcomes in long-standing type 1 diabetes: a longitudinal follow-up. Diabetes Care. 2018;41(12):2487-94. 
47. Feodoroff M, Harjutsalo V, Forsblom C, Groop PH, FinnDiane Study Group. Dose-dependent effect of smoking on risk of coronary heart disease, heart failure and stroke in individuals with type 1 diabetes. Diabetologia. 2018;61(12):2580-9.

48. Pearce I, Simó R, Lövestam-Adrian M, Wong DT, Evans M. Association between diabetic eye disease and other complications of diabetes: implications for care. A systematic review. Diabetes Obes Metab. 2019;21(3):467-78.

49. Guo VY, Cao B, Wu X, Lee JJW, Zee BC. Prospective association between diabetic retinopathy and cardiovascular disease-a systematic review and meta-analysis of cohort studies. J Stroke Cerebrovasc Dis. 2016;25(7):1688-95.
50. Jansson FJ, Forsblom C, Harjutsalo V, Thorn LM, Wadén J, Elonen N, et al. FinnDiane Study Group. Regression of albuminuria and its association with incident cardiovascular outcomes and mortality in type 1 diabetes: the FinnDiane Study. Diabetologia. 2018;61(5):1203-11.

\section{Publisher's Note}

Springer Nature remains neutral with regard to jurisdictional claims in published maps and institutional affiliations.
Ready to submit your research? Choose BMC and benefit from:

- fast, convenient online submission

- thorough peer review by experienced researchers in your field

- rapid publication on acceptance

- support for research data, including large and complex data types

- gold Open Access which fosters wider collaboration and increased citations

- maximum visibility for your research: over $100 \mathrm{M}$ website views per year

At BMC, research is always in progress.

Learn more biomedcentral.com/submissions 